\title{
PROPRIEDADES AVERSIVAS EM CONTINGÊNCIAS DE REFORÇAMENTO POSITIVO: EVIDÊNCIAS EMPÍRICAS
}

\section{AVERSIVE PROPERTIES IN POSITIVE REINFORCEMENT CONTINGENCIES: EMPIRICAL EVIDENCE}

\author{
FERNANDA BORDIGNON LUIZ - ORCID 0000-0002-6878-6252 \\ UNIVERSIDADE dE SÃo PAULO E UNIVERSIDADE POSITIVO, BRASIL \\ MARIA HelenA LeITE HUNZIKER - ORCID 0000-0003-0030-375K \\ UNIVERSIDADE DE SÃO PAULO, BRASIL
}

\section{RESUMO}

A divisão binária entre reforçamento positivo e controle aversivo estabelece que os dois tipos de controle são excludentes entre si. Em direção contrária, alguns autores vêm defendendo que essa dicotomia não se sustenta uma vez que contingências de reforçamento positivo podem envolver também funções aversivas. O presente trabalho teve por objetivo sistematizar alguns dos experimentos presentes na literatura que fundamentam essa proposição, agrupando-os em três conjuntos, de acordo com o tema central do estudo: pausa pós-reforço, autoimposição de time-out e indução de comportamento agressivo. Como resultado, pode-se demonstrar que pesquisas sobre três temas distintos levam a uma mesma conclusão: a distinção binária controle aversivo/reforçamento positivo não descreve adequadamente as relações de controle do comportamento. No nível teórico/conceitual, tal proposição fortalece a necessidade de revisão sobre o que se convencionou denominar controle aversivo ou controle por reforço positivo. No nível aplicado, em que geralmente são destacados os efeitos indesejáveis apenas de contingências aversivas, essa proposição tem implicações éticas em relação ao uso de contingências que controlam o comportamento, individual ou social, independentemente da sua caracterização como reforçadoras positivas ou aversivas

Palavras-chave: controle aversivo; reforçamento positivo; pausas pós-reforço, autoimposição de time-out, comportamento agressivo.

\begin{abstract}
The binary division between positive reinforcement and aversive control establishes that the two types of control are mutually exclusive. However, some authors have argued that this dichotomy does not stand because contingencies of positive reinforcement may also involve aversive functions. The present study aimed to systematize some of the experiments in the literature that support this proposition. We organized them into three sets according to the central theme of the study: post-reinforcement pause, self-imposed time-out, and induction of aggressive behavior. As a result, research on three distinct themes can be shown to lead to the same conclusion: the aversive control/positive reinforcement binary distinction does not adequately describe behavior control relationships. At the theoretical/conceptual level, this proposition strengthens the need to review what has been termed as aversive control or control by positive reinforcement. At the applied level, in which the undesirable effects of aversive contingencies are usually highlighted, this proposition has ethical implications regarding the use of contingencies that control individual or social behavior, regardless of their characterization as positive or aversive reinforcers. aggression.

Key words: aversive control; positive reinforcement; post-reinforcement pauses; self-imposed time-out;

\footnotetext{
Este trabalho foi desenvolvido como parte da tese de doutorado que está sendo realizada pela primeira autora, sob orientação da segunda, no Programa de Pós-Graduação em Psicologia Experimental da USP. Foi financiado pelo CNPq com Bolsa de Doutorado (processo número 142293/2017-2) e Bolsa de Produtividade em Pesquisa (processo número 304120/2016-2). Correspondência referente a este artigo deve ser endereçada a Fernanda Bordignon Luiz (febluiz@gmail.com).
}

DOI. 10.18542/rebac.v14i2.7535 
As contingências que controlam o comportamento vêm sendo comumente agrupadas em duas grandes categorias: as que utilizam reforço positivo e as que envolvem controle aversivo (neste caso, punição positiva, punição negativa e reforçamento negativo - ver Catania, 1999). Contudo, essas duas categorias não têm recebido a mesma atenção nas últimas décadas: estudos recentes mostram que a partir dos anos 1970 houve enorme concentração nas investigações sobre contingências utilizando reforço positivo, sendo minoritários os estudos envolvendo contingências comumente conhecidas como parte do controle aversivo (Catania, 2008; Rico, Carvalho Neto, Silveira, \& Barros, 2018). Isso significa que a Análise do Comportamento vem sendo desenvolvida de forma desequilibrada em relação aos temas pesquisados, se consideradas as duas classes apontadas.

Apesar desse desequilíbrio, a divisão binária tem sido mantida e utilizada para apontar aspectos considerados indesejáveis em relação às contingências aversivas, mas não às de reforçamento positivo. Por exemplo, Skinner, ao longo de sua obra, defendeu que a punição é uma técnica ineficaz por produzir efeitos indesejáveis e temporários de supressão do responder (Skinner, 1953; 1971; 1974, entre outros), enquanto Sidman (1989) associou o controle aversivo à noção de coerção ao afirmar que "O controle por reforçamento positivo não é coercitivo; a coerção entra em cena quando nossas ações são controladas por reforçamento negativo ou punição" (p. 43). Nesse contexto, criou-se um quase consenso de que o controle por reforçamento positivo é condição para o estabelecimento de comportamentos individual e socialmente desejáveis/relevantes, enquanto que o controle aversivo seria prejudicial a quem está submetido a ele e, por isso, deve ser evitado por questões éticas. Como consequência, as prescrições de uso de diferentes contingências em situações de aplicação têm enfatizado os prejuízos decorrentes do uso do controle aversivo, com pouca análise sendo feita sobre os problemas decorrentes do uso do reforçamento positivo (Campos, 2010)

Alguns pesquisadores têm sido vozes destoantes, tanto em nível teórico (Balsam \& Bondy, 1983; Perone, 2003) como em situações voltadas ao atendimento clínico (Guilhardi, 2016; Banaco \& Zamignani, 2018) ou ao planejamento cultural (Critchfield, 2014). Em relação a questões conceituais, foram apontadas algumas dificuldades na distinção entre os dois tipos de controle (Michael, 1975; Hineline, 1984; Baron \& Galizio, 2005; Hunziker, 2011), sugerindo-se que a questão binária (aversivo versus positivo) ainda está aberta, necessitando de maiores investigações a respeito.

No nível experimental, estudos realizados sobre diferentes temas têm apresentado dados que sugerem que essa divisão binária não é adequada para se compreender o controle do comportamento, uma vez que foram identificados processos aversivos como função de contingências tradicionais de reforçamento positivo. Considerando que a Análise do Comportamento avança com base em evidências experimentais, o presente artigo teve por objetivo realizar uma revisão sobre esses estudos, utilizando como estratégia seu agrupamento de acordo com o tema central investigado, a saber a) pausas pósreforço, b) autoimposição de time-out, e c) comportamentos agressivos. Conforme poderá ser visto a seguir, essa sistematização dos estudos nas três temáticas viabiliza uma dimensão ampla do questionamento sobre a dicotomia entre o que se convencionou denominar controle aversivo e controle por reforço positivo.

\section{ESTUDOS SOBRE PAUSA PÓS-REFORÇO POSITIVO}

As pausas no responder foram incialmente identificadas por Ferster e Skinner (1957) em seus estudos sobre esquemas de reforçamento positivo em razão fixa ${ }^{1}$. Os autores demonstraram como padrão típico de comportamento a alta frequência de respostas até a obtenção do reforço, seguida de ausência de respostas por algum tempo, denominada "pausa"; terminada essa pausa, ocorre transição relativamente rápida para alta frequência de respostas até a produção do próximo reforçador, com nova pausa, e assim sucessivamente. Esse tempo de interrupção do responder, ocorrendo em seguida a liberação do reforço, é denominado de "pausa pósreforço". Em função da lógica do esquema de razão, quanto mais o sujeito responder mais ele ganha. Portanto, a pausa pós-reforço não parece funcional, pois ela tem como consequência adiar o acesso ao próximo reforço, além de diminuir a quantidade de reforçadores recebidos na sessão (se ela tiver duração fixa). A questão que se colocou logo que esse dado foi relatado dizia respeito a essa funcionalidade do comportamento: se o sujeito perde reforços fazendo as pausas, por que ele as apresenta? Em outras palavras, o que controla a ocorrência das pausas pós-reforço?

Ferster e Skinner (1957) investigaram a relação entre a quantidade de respostas exigidas no esquema de razão fixa e a duração da pausa pós reforço. Em um dos experimentos, pombos foram expostos a um esquema de FR1 aumentado gradativamente até FR 120. Os resultados sugeriram que quanto maior a razão do esquema, maior a duração das pausas que ocorreram após os reforços. Outros experimentos confirmaram que a razão utilizada para liberação do reforço é uma variável que interfere na duração da pausa (Felton \& Lyon, 1966; Powell, 1968).

Griffiths e Thompson (1973) ampliaram essa análise ao investigar a importância não apenas do tamanho da razão, em si, mas da relatividade da razão a ser cumprida no momento atual em comparação com razões anteriores. Para isso, eles investigaram, com ratos, a duração das pausas em esquemas múltiplos e mistos ${ }^{2}$ que envolviam razões altas e baixas. Foram medidas as pausas apresentadas após a liberação do reforço, analisando-as em função das características dos componentes anteriores

\footnotetext{
1 No qual a apresentação de um reforçador se dá após a emissão de uma determinada quantidade fixa de respostas.

${ }^{2}$ Esquema múltiplo envolve mais de um esquema alternando-se, cada um acompanhado por sinalização diferenciada. Esquema misto é semelhante ao anterior, porém sem sinalização (Catania, 1999).
} 
aqueles em vigor: a) razão alta após razão baixa; b) razão baixa após razão alta; c) razão alta após razão alta e d) razão baixa após razão baixa. A designação de baixa e alta era decorrente da relação entre elas, ou seja, a razão baixa era correspondente a $x$, enquanto a alta correspondia a $2 x$, sendo, nesse estudo, $x=20,30$ e 60 , respectivamente para cada um dos três sujeitos. Os resultados demonstraram que, nos esquemas mistos, a duração das pausas não diferiu significativamente nas quatro possíveis transições entre razões. Já nos esquemas múltiplos, as pausas foram mais extensas (30s ou mais) antes dos componentes de razão alta, sendo ainda maior quando a razão alta era precedida por um componente de razão baixa. Uma vez que a diferença entre os esquemas múltiplo e misto é apenas a presença (ou não) do estímulo correlacionado ao esquema em vigor, esses resultados sugeriram que a pausa dos animais estava sob controle do estímulo que sinalizava a razão que iria vigorar no componente que iniciava após o reforço e sua relação (maior, menor ou igual) com a razão que vigorou no componente anterior ${ }^{3}$.

A demonstração de que a pausa pós-reforço pode ser função da interação entre diferentes componentes de um esquema múltiplo passou a nortear novos estudos. Baron e Herpolsheimer (1999) realizaram um experimento, com ratos, no qual os componentes foram variados e denominados de "baixo" e "alto" valor (relativizada pela razão alternativa). O componente de baixo valor (FR 20) foi mantido constante e foi variada a razão do componente alternativo (FR 30 a FR 150). Os resultados indicaram que quanto maior a diferença entre os valores da razão de cada componente, maior a duração da pausa antes do componente de alta exigência. Ou seja, as pausas ocorreram com maior duração após a liberação do reforço da razão de menor exigência e antes da primeira resposta no componente de maior exigência (período de transição ${ }^{4}$ entre componentes).

Em outro estudo, com pombos, Perone e Courtney (1992) manipularam diferentes magnitudes do reforço (duração de acesso a grãos) denominando os componentes como "rico" ou "pobre", em esquemas múltiplos e mistos, mantendo constante a razão de cada componente. Os resultados indicaram que quanto maior a diferença entre a magnitude dos reforçadores entre os componentes, pausas mais extensas ocorreram na transição entre o componente mais favorável para o menos favorável, porém, esse padrão foi produzido apenas

\footnotetext{
3 O termo "pausa pós-reforço" sugere que a pausa é controlada pelo reforço que a antecede (Ferster \& Skinner, 1957). Após a demonstração de que esse controle depende de uma relação entre as razões sucessivas, e aos estímulos que as controlam, outros termos foram sugeridos como possivelmente mais apropriados, tal como "pausa entre razões" (between-ratio pause) ou pausa pré-razão (Griffiths \& Thompsom, 1964). Contudo, o termo original "pausa pós-reforço" continua sendo usado em publicações recentes (exemplo, Young, Foster, \& Bizo, 2017) e, por isso, será mantido aqui.

${ }^{4} \mathrm{O}$ termo transição está sendo entendido como o período entre o final de um componente (liberação do reforço) e a primeira resposta do novo componente (Perone, 2019).
}

quando tais componentes eram sinalizados, ou seja, no esquema múltiplo, mas não no misto.

A relação entre os componentes também foi medida em estudo que manipulou a força necessária para pressionar a barra (Wade-Galuska, Perone, \& Wirth, 2005). Utilizando ratos como sujeitos em um esquema múltiplo com duas barras, os autores mediram as pausas entre componentes que exigiam diferentes intensidades de força de pressão à barra $(0.25 \mathrm{~N}$ e $0.15 \mathrm{~N})$, nos quais apenas umas delas estava disponível (sinalizando qual o componente estava em vigor). Os autores identificaram que pausas mais longas ocorreram na transição entre o componente que exigia menor força de pressão à barra para o que exigia maior força.

A pausa pós-reforço também foi estudada em função do atraso do reforço positivo. No experimento de Harris, Foster, Levine e Temple (2012) foram utilizadas galinhas como sujeitos e manipulados valores de atraso do reforço $(0 \mathrm{~s}, 4 \mathrm{~s}, 8 \mathrm{~s}, 16 \mathrm{~s}$ e $32 \mathrm{~s})$ em um dos componentes de um esquema múltiplo, com razão fixa. Os resultados mostraram que quando há estímulo correlacionado com o componente com reforço atrasado, as pausas são mais longas nas transições entre um componente com reforço imediato para o componente com atraso de reforço. No estudo de Young, Foster e Bizo (2017), também realizado com galinhas, foram manipuladas diferentes magnitudes de reforço (1s ou 6 s de acesso a grãos) em esquemas múltiplos, mistos e simples. Uma das principais diferenças entre esse estudo e o de Perone e Courtney (1992) foi, além do sujeito experimental, o uso de diferentes razões apresentadas parametricamente (FR 1 a FR 128) de modo a mensurar os efeitos das diferentes magnitudes de reforço, em diferentes razões. Os resultados de Young et al. (2017) mostraram que as pausas eram maiores durante componentes de maior razão, obtendo-se, nos esquemas múltiplos, a mesma relação anteriormente apontada: as maiores pausas ocorreram após a liberação dos reforços em componentes de maiores magnitudes de reforço e antes da primeira resposta no componente de menor magnitude de reforço. A diferença entre a magnitude do reforço de cada componente exerceu maior controle para o comportamento de pausar em esquemas de maior razão. Em esquemas mistos, o comportamento das galinhas ficou mais sob controle da magnitude do último reforço recebido, apresentando menores pausas após reforçadores de menor magnitude. Nos esquemas simples, não houve diferença significativa do efeito da magnitude do reforço sobre a duração das pausas em função do tamanho da razão (FR 32, FR 4, FR 16).

Em resumo, os estudos com animais não humanos mostraram que a pausa é função não apenas da razão do esquema em vigor, como também da sua relação com o componente que o antecedeu. Utilizando-se sujeitos humanos, a relação observada tem sido semelhante. Por exemplo, Williams, Saunders e Perone (2011) realizaram dois experimentos (um múltiplo e um misto) com adultos com deficiência intelectual leve, nos quais foram manipulados diferentes requerimentos de resposta e magnitude do reforço: o componente denominado "rico" exigia menos respostas e liberava maior quantidade de 
reforço - dinheiro - do que o componente "pobre". No estudo em que o esquema utilizado foi o múltiplo, os resultados foram os mesmos anteriormente descritos em estudos com sujeitos não humanos: as maiores pausas ocorreram na transição entre o componente rico para o pobre. No esquema misto, não houve pausas extensas. Portanto, com humanos ou não humanos, os estudos sobre pausas em esquemas de reforçamento positivo produziram resultados bastante regulares quando foram utilizados esquema múltiplos (mas não esquemas em mistos), sugerindo que o comportamento de pausar seja controlado pelo estímulo que sinaliza o esquema que irá vigorar em seguida.

Uma das interpretações possíveis sobre esse conjunto de dados é a de que o estímulo correlacionado ao menor ganho, comparativo à condição anterior, adquire função aversiva suprimindo temporariamente o responder (para uma revisão sobre estudos da pausa em esquemas de reforçamento, ver Schlinger, Derenne, \& Baron, 2008). Ou seja, nessa interpretação, a função aversiva desse estímulo é adquirida no contexto comparativo entre duas ou mais condições de reforçamento positivo que estabelecem diferentes relações custo/benefício. Isso equivale a dizer que pode haver diferentes graus de aversividade em esquemas de reforçamento positivo, a depender das características das variáveis que os constituem.

\section{AUTOIMPOSIÇÃO DE TIME-OUT ${ }^{5}$}

O segundo conjunto de dados que sugerem a presença de aversividade em contingências de reforçamento positivo é constituído por estudos que mediram a autoimposição de time-out (TO), ou seja, o indivíduo apresenta uma resposta que suspende a contingência de reforçamento positivo em vigor e os estímulos a ela correlacionados.

O primeiro estudo dessa linha de investigação foi realizado por Azrin (1961), que treinou pombos em caixa experimental contendo duas chaves: bicar uma delas produzia reforço (alimento) sob diversos esquemas simples de reforçamento em razão fixa, que variavam entre FR 50 a FR 200; bicar a segunda chave desligava as luzes da caixa e suspendia a contingência de reforçamento positivo em vigor (TO). Nova bicada nessa mesma chave restaurava as luzes e o esquema de reforçamento voltava a vigorar na chave alternativa. Portanto, além de ter controle sobre a apresentação do alimento bicando uma das chaves, o pombo também podia controlar a ocorrência e a duração do TO bicando a chave alternativa. Os resultados

\footnotetext{
${ }^{5} \mathrm{O}$ termo TO tem sido utilizado na literatura com diversos significados. Leitenberg (1965) afirma que o principal aspecto que o caracteriza é um período de tempo no qual o reforço positivo não é disponibilizado. Tal definição englobaria a extinção, o estímulo delta em treinos discriminativos e também os intervalos entre reforços em esquemas intermitentes. Nos estudos caracterizados neste artigo, autoimposição do TO referese à resposta do próprio sujeito que produz a suspensão da contingência de reforço positivo, além de produzir a apresentação de estímulo associado a esse período de extinção.
}

mostraram que os sujeitos apresentaram bicadas na chave consequenciada com alimentos mostrando o padrão típico de esquemas de razão fixa (Ferster \& Skinner, 1957). Além disso, ficou também demonstrado que os pombos bicaram a chave que produzia $\mathrm{TO}$, fazendo isso com maior frequência logo após terem obtido o reforço na chave alternativa. A manipulação do tamanho da razão para liberação do reforço positivo permitiu identificar que a duração do TO foi função direta do tamanho da razão requerida para obter o alimento: quanto mais alta a razão para produção de alimento, maior a duração do TO autoimposto. Se no esquema de razão quanto mais o animal responde mais ele ganha, por que o pombo bica a segunda chave se isso adia a obtenção do próximo reforço? Essa questão é muito parecida com a formulada em relação à pausa pós-reforço, com o diferencial de que aqui o animal apresenta uma resposta ativa (bicar a segunda chave) e não apenas deixa de responder, como nas pausas. Dado que a única consequência de bicar essa segunda chave era cancelar temporariamente o funcionamento do esquema de reforçamento positivo e remover os estímulos a ele correlacionados, interpretou-se que a resposta de bicar essa chave estaria sendo reforçada negativamente, o que a caracterizaria como resposta de fuga, e o momento pós-reforço positivo (e os estímulos correlacionados a esse esquema) teria a função de estímulo aversivo. Em outras palavras, um esquema de reforçamento positivo envolvendo propriedades aversivas.

O experimento pioneiro de Azrin (1961) foi seguido por outros (Thompson, 1964; 1965) que replicaram a demonstração de que animais impõem a si mesmos interrupções de esquemas de reforçamento positivo em razão fixa. No estudo de Thompson (1964) foram realizados experimentos, com ratos, utilizando duas barras, uma relacionada à liberação do reforço positivo em razão fixa, e outra cuja pressão produzia TO de duração fixa de 30 segundos; após esse período, nova pressão a essa barra restaurava a contingência de reforçamento positivo. No primeiro experimento, foi utilizado um esquema simples no qual a razão requerida para reforçamento aumentou gradativamente de 25 em 25 respostas, iniciando com FR 25 até uma razão máxima que produzisse pausas com duração maior que 50 segundos (chegou-se a FR 250). Após atingir essa razão máxima, a exigência foi diminuindo de 25 em 25 respostas novamente, até retornar ao valor inicial (FR 25). Obtevese que a frequência de produção de TO foi maior em razões mais exigentes do que em esquemas menos exigentes, tendo ocorrido principalmente no período pósreforço. Em outro experimento, um sujeito foi submetido a um esquema misto FR 25 FR 225. Os resultados mostraram que o sujeito produziu $\mathrm{TO}$ apenas no componente de maior exigência (FR 225), sendo a sua ocorrência mais provável após pelo menos 25 respostas. Interpretou-se que, ainda que os esquemas não fossem sinalizados, a diferença entre as duas razões era tamanha que o FR225 passava a ser previsível após a $25^{\mathrm{a}}$ resposta não ser seguida de reforço. No terceiro experimento, foi aumentada a exigência para a produção de TO: de FR 3 até FR 27 na barra do TO, mantido fixo o valor de FR 475 na 
barra associada ao reforço positivo. Os resultados mostraram que o sujeito respondeu na barra de TO até atingir FR 15, deixando de apresentar essa resposta quando as razões foram maiores. Dessa forma, ficou demonstrado que o TO pode ser uma consequência de alto valor reforçador dado que o animal chegou a emitir 15 respostas para produzi-lo.

As similaridades entre os resultados dos estudos de autoimposição de TO com os descritos nos estudos sobre pausa pós-reforço têm fortalecido a interpretação de que ambos (pausas e TO) são comportamentos de fuga controlados por propriedades aversivas inerentes ao esquema de reforçamento positivo. Poucos estudos questionaram essa interpretação. Um deles foi o de Appel (1963), no qual a bicada do pombo em uma das chaves era reforçada positivamente em diferentes esquemas de reforçamento de razão fixa, enquanto bicadas na segunda chave podiam produzir uma dentre três diferentes consequências: (a) modificar os estímulos visuais (apagavam-se as luzes da caixa e modificavam a cor da chave de ambos os discos) e eliminar a contingência de reforçamento positivo; (b) modificar os estímulos visuais e não modificar a contingência de reforçamento positivo; (c) não produzir nenhuma mudança. Uma segunda resposta nessa chave restaurava a condição inicial nas condições (a) e (b). Os animais foram dispostos em grupos, cada um submetido a uma dessas condições. Obteve-se como resultado que na condição (c) os pombos raramente bicavam a segunda chave. Contudo, quando a bicada na segunda chave produzia mudança nos estímulos visuais, suspendendo ou não a contingência de reforçamento positivo - condições (a) e (b) - a quantidade de bicadas nessa chave ocorreu em função da razão requerida na chave alternativa. Portanto, para Appel, a suspensão da possibilidade de reforço não foi a variável que exerceu controle no bicar a chave que produzia o TO. Ele interpretou esses resultados como indicadores de que a mudança do estímulo correlacionado ao esquema é, em si, um estímulo reforçador positivo, não sendo necessária a suposição das funções aversivas sugeridas por Azrin (1961).

Apesar de o estudo de Appel (1963) ser instigante por contrariar os demais - por não confirmar a interpretação de que a autoimposição de TO é uma resposta de fuga do esquema de reforçamento positivo não encontramos nenhuma replicação desse procedimento, o que sugere que ele não teve grande influência na área. Assim, com apenas essa exceção, a interpretação acerca do TO autoimposto como sendo uma resposta de fuga é a aceita nos estudos sobre esse tema: foi essa a interpretação defendida no primeiro trabalho de revisão dessa área (Leitenberg, 1965), e é a que se mantém nos trabalhos mais recentes (Perone, 2003; Everly, Holtyn, \& Perone, 2014; Retzlaff, Parthum, Pitts, \& Hughes, 2017).

Outras variáveis foram analisadas nos procedimentos de estudo sobre a autoimposição do TO. Resultados contraditórios foram relatados quando a duração do TO não ficou sob controle do sujeito: Zimmerman e Ferster (1964) obtiveram, com pombos, baixa produção de TO com duração fixa (10s), enquanto
Thompson (1964; 1965) obteve autoimposição de TO com duração fixa de 30s, tanto com ratos quanto com pombos. Outros esquemas de reforçamento positivo foram empregados, tais como esquema de reforçamento diferencial em baixas taxas (DRL, differential reinforcement of low rates) (Lydersen, 1992), tempo fixo (FT) (Lydersen, 1997), intervalo fixo (FI) (Brown \& Flory, 1972; Cohen \& Campagnoni, 1989) e razão progressiva (PR) (Dardano, 1973).

Um estudo mediu as funções de estímulos que sinalizam os componentes em esquemas múltiplos de reforçamento, com ênfase nas transições entre o mais para o menos vantajoso em termos de magnitude do reforçador (Everly, Holtyn, \& Perone, 2014). Foi estabelecido um procedimento no qual os sujeitos poderiam desligar o estímulo que sinalizava a magnitude do próximo reforçador. Os resultados indicaram que todos os pombos bicavam mais frequentemente a chave que removia o estímulo correlacionado ao esquema de menor magnitude de reforço. Metade dos pombos respondeu em função não apenas da sinalização da magnitude do próximo reforçador, mas também da magnitude do reforçador anterior. Esse resultado foi interpretado como fortalecedor da hipótese de controle aversivo exercido pelo estímulo correlacionado ao reforçador positivo de menor magnitude. Retzlaff et al. (2017, Exp. 2) também realizaram um experimento com pombos, no qual foi disponibilizada uma chave que produzia TO nos moldes relatados por Azrin (1961). Foram utilizados quatro estímulos diferentes (cores das luzes dos discos) para diferenciar os quatro tipos de transição entre componentes. Obteve-se que, para a maioria dos pombos, a produção de TO foi mais provável entre a transição do reforço mais vantajoso (alta magnitude) para o menos vantajoso (baixa magnitude).

Em conjunto, os estudos de autoimposição de TO sugerem que, frente a esquemas múltiplos de reforçamento positivo, a autoimposição de TO será tanto maior quanto maior for a diferença entre os componentes, em especial se houver transição de uma contingência "mais vantajosa" para outra "menos vantajosa". Essa relação parece indicar que pode haver diferentes graus de aversividade em esquemas de reforçamento positivo em FR. Além disso, os dados apontam que o momento logo após a liberação do reforço é, aparentemente, o mais aversivo.

\section{COMPORTAMENTOS AGRESSIVOS SOB REFORÇAMENTO POSITIVO}

Os estudos sobre agressão eliciada por estímulos que geralmente desempenham função de aversivos ao integrar contingências de punição positiva e reforçamento negativo (como o choque elétrico) fundamentam que se considere aversivo todo o estímulo que induza/elicie comportamentos agressivos (Ulrich \& Azrin, 1962; Azrin, Hutchinson, \& Hake, 1963; Ulrich, Wolff, \& Azrin, 1964; Azrin, Hake, \& Hutchinson, 1965; Azrin, Hutchinson, \& McLaughlin, 1965; Ulrich, 1966). É nesse contexto que deve ser considerado o terceiro conjunto de estudos aqui apresentado: a ocorrência de comportamentos agressivos 
induzidos por esquema de reforçamento positivo, sugerindo que esses esquemas têm componentes aversivos. Em estudos com animais não humanos, o comportamento de agredir é caracterizado topograficamente, envolvendo contato físico do agressor em direção ao agredido (também chamado de "alvo"), com a produção de danos físicos. No caso de pombos, a agressão corresponde a sequências de bicadas; em mamíferos (ratos ou macacos), as mordidas direcionadas ao "alvo". Nesses arranjos, geralmente o animal "alvo" encontra-se em restrição física, que o impede de reagir, protegido por uma capa para evitar danos físicos. ${ }^{6}$

Novamente de forma pioneira, Azrin, Hutchinson e Hake (1966) realizaram experimentos com o objetivo de avaliar se o reforço positivo poderia produzir agressão. Para tanto, pombos foram submetidos inicialmente a um período de não reforçamento, para medir a linha de base do comportamento de agredir. Em seguida, foram expostos a sequências de reforçamento positivo contínuo (CRF) seguido por cinco minutos de extinção. Os resultados mostraram que todos os pombos atacaram mais o alvo durante o período de extinção seguido de reforçamento contínuo do que durante o período de não reforçamento (linha de base). Em outros experimentos, os autores manipularam diferentes variáveis, como liberação do alimento de forma livre, sinalização da extinção, tipo de alvo (vivo ou inanimado) e quantidades de reforços disponibilizados durante o período de reforçamento. Os dados de todos esses experimentos evidenciam que o momento em que há maior frequência de ataques é o de transição do reforçamento contínuo para a extinção.

A partir desse estudo de Azrin et al. (1966), vários outros foram realizados, com diferentes tipos de esquemas de reforçamento positivo. Utilizando esquema de FR, Gentry (1968) mediu a quantidade de agressão de pombos submetidos a um delineamento $\mathrm{ABAB}$, onde a condição A envolvia ausência de reforçamento e condição $\mathrm{B}$ a reforçamento em FR 50. Os resultados mostraram que, assim como descrito por Azrin et al. (1966), os ataques ocorreram apenas na codição $\mathrm{B}, \operatorname{logo}$ após o consumo do reforço, não sendo registrada ocorrência de agressão na condição A. Com base nesses dados, foi fortalecida a suposição de que a intermitência do reforço positivo contém propriedades aversivas.

Knutson (1970), por sua vez, estendeu as descobertas anteriores ao medir agressão de animais expostos a esquemas com diferentes valores de FR. Foram utilizadas diferentes combinações de valores de FR em esquemas múltiplos (FR $x-$ EXT - FR $1-$ EXT, sendo $x=$ $1,15,25,40,60$ ou 120). Os resultados mostraram que em componentes de menor valor de FR (FR 1, 15 e 25), não ocorreu significativa quantidade de agressão, ao contrário do obtido sob esquemas de valor comparativamente alto (FR 40, 60 e 120).

Knutson e Kleinknecht (1970) realizaram um estudo com quatro pombos submetidos à sessões de 60

\footnotetext{
${ }^{6}$ Sobre estudos relativos à agressão eliciada, ver revisão de Soares e Goulart (2015) e Soares e Carvalho Neto (2016).
}

reforços em esquema FR 1, seguidos de sessões com reforço diferencial de baixas taxas (DRL 20s) e, por fim, novamente a sessões de FR 1. Os resultados revelaram que nas sessões de FR 1 não houve ocorrência de agressão. Durante as sessões em DRL 20, todos os pombos apresentaram respostas de agressão, que também ocorreram no início da nova sessão de FR 1, cessando em seguida. Considerando que tanto no esquema DRL 20 quanto em FR 120 a densidade de reforço é baixa, e que sob esses dois esquemas houve maior ocorrência de agressão em comparação a outros esquemas, considerou-se que a densidade de reforço positivo pode ser uma variável relevante para a indução do comportamento agressivo.

Comportamentos agressivos foram também estudados sob diversos esquemas de intervalo envolvendo reforçamento positivo. Cherek e Heistad (1971) utilizaram pombos em um delineamento $\mathrm{ABAB}$ (condição $\mathrm{A}$, sem reforçamento e condição B em FI $60 \mathrm{~s}$ ). Os ataques ao alvo ocorreram durante as sessões de FI, principalmente após o reforçamento e no início do intervalo. Os ataques diminuíram à medida que o tempo do intervalo passava e se aproximava o próximo reforço.

Pitts e Malagodi (1996) compararam esquemas de razão e intervalo utilizando um esquema múltiplo de intervalo fixo (FI) e razão fixa (FR). No esquema de intervalo, o disco era iluminado na cor branca e a primeira bicada após transcorrido $t$ minutos (4 minutos para dois pombos e 12 minutos para outro) tornava o disco vermelho e entrava em vigor o esquema de razão FR 1 x $n$, sendo $n$ iniciando com 1 e aumentando para 8, 16 e 24 . Os resultados mostram que nenhum ataque ocorreu durante o período em que o reforço estava disponível, porém muitos ocorreram no período logo após o encerramento da disponibilização do reforço. Esses dados fortaleceram a noção de que a aversividade é inerente ao período pós liberação do reforço positivo, sendo função direta da magnitude do reforço recebido.

De maneira geral, nos diferentes estudos um resultado consistente é o de que as respostas agressivas ocorrem logo após a disponibilização do reforço em esquemas intermitentes. Considerando que os esquemas contínuos são raros no contexto natural dos organismos, esses dados sugerem que as contingências de reforçamento positivo geralmente incluem, em diferentes graus, componentes aversivos.

\section{CONCLUSÃO}

Os três conjuntos de experimentos apresentados demonstram que, ao agruparmos pesquisas sobre temas tão distintos, pode-se fundamentar experimentalmente uma proposição geral que contraria a forma dicotômica de controle positivo versus aversivo. Esse conjunto de dados sugere fortemente a não linearidade da distinção entre as relações que envolvem reforço positivo e as que envolvem contingências ditas aversivas (punição positiva/negativa e reforçamento negativo, indicando que ambas as relações podem coexistir em uma mesma contingência. Além do questionamento teórico/conceitual, esses dados colocam em cheque as prescrições (também dicotômicas) sobre o uso de ambos os controles no nível individual ou social 
aplicado. Essa perspectiva não dualista fortalece a noção de que uma contingência não é, em si, "boa" ou "ruim" sendo sua função dependente, entre outras variáveis, das contingências alternativas disponíveis no momento ou experimentadas anteriormente pelo sujeito (Perone, 2003). Tal perspectiva exige análises comportamentais amplas, que levem em conta o conjunto de contingências no qual o sujeito está (ou esteve) inserido e não apenas que seja considerada a contingência atual em estudo.

A análise sobre possíveis efeitos "negativos" (no sentido de prejudiciais ao sujeito) de contingências de reforçamento positivo parece apontar na direção de que o reforçamento positivo pode ter mais similaridades com algumas contingências denominadas aversivas do que geralmente se supõe. Revisando a literatura sobre efeitos respondentes e operantes de contingências de reforçamento positivo, Balsam e Bondy (1983) concluíram que eliciação de respostas incompatíveis com a respostaalvo, bem como seleção de comportamentos indesejáveis, podem ocorrer tanto sob reforçamento positivo como sob punição. Portanto, a adequação de uma contingência em contexto aplicado não está no fato de ela ser "aversiva" ou "não aversiva", mas sim depende da precisão da análise efetuada, que deve abranger todo o contexto atual e histórico do sujeito.

Após analisar diversos estudos experimentais, Perone (2003) conclui que componentes aversivos são inevitáveis em todas as relações, mesmo aquelas que envolvem apenas componentes de esquemas de reforçamento positivo. Nessa direção, Hunziker (2017) acrescenta que mesmo em esquemas de reforçamento contínuo há a necessidade de estabelecimento de algum grau de aversividade, dado que a função reforçadora de um estímulo depende da sua privação (que é aversiva); também destaca que uma história de reforçamento positivo, quando interrompida (extinção), pode estabelecer uma condição extremamente aversiva que não ocorreria se não houvesse esse histórico de reforçamento. Ou seja, olhando por diferentes ângulos, a conclusão parece ser a mesma: a dicotomia é falsa; a aversividade é parte inerente do controle do comportamento e ocorre de forma interligada com o reforçamento positivo. Variações nos arranjos de contingências podem influenciar a magnitude da sua aversividade: características do antecedente ou do consequente, outras contingências paralelas, história dos sujeitos, entre outras. Essa multiplicidade de variáveis nos obriga a uma análise aprofundada, de amplo espectro, que evite, a priori, a condenação do uso de contingências ditas aversivas ou a aceitação daquelas que supostamente utilizam apenas reforço positivo (Critchfield, 2014).

A literatura nos mostra que ainda estamos longe de compreender a complexidade dos controles sobre o comportamento. Especificamente, esse conjunto de estudos sugere que a divisão binária entre controles aversivo versus positivo é limitante, favorecendo um entendimento truncado, simplista e muitas vezes "dogmático" sobre o comportamento, que desvia a atenção da multiplicidade de controles existentes (Hunziker, 2017). Esses estudos fortalecem a análise de Hineline (1984) de que o controle aversivo não é uma área do saber separada daquela que estuda o controle por reforçamento positivo. Referenciando Perone (2003), podemos considerar que "nossa preocupação não deveria ser se a contingência envolve reforçamento positivo, negativo ou punição, em vez disso, devemos enfatizar a capacidade das contingências para fomentar o comportamento de interesse ao indivíduo em longo prazo" (p. 13). As evidências produzidas pelos experimentos aqui apresentados fortalecem essa sugestão.

\section{DECLARAÇÃO DE CONFLITO DE INTERESSES}

As autoras declaram que não há conflito de interesses relativos à publicação deste artigo e certificam que ambas participaram do trabalho, sendo igualmente responsáveis pelo seu conteúdo.

\section{CONTRIBUIÇÃO DE CADA AUTORA}

Ambas as autoras atuaram nas etapas de escolha temática, concepção do artigo e redação do texto final. F. Bordignon-Luiz realizou, adicionalmente, o levantamento e organização dos estudos aqui apresentados.

\section{DIREITOS AUTORAIS}

Este é um artigo aberto e pode ser reproduzido livremente, distribuído, transmitido ou modificado, por qualquer pessoa desde que usado sem fins comerciais. O trabalho é disponibilizado sob a licença Creative Commons 4.0 BY-NC.

\section{(cc) BY-NC}

\section{REFERÊNCIAS}

Appel, J. B. (1963). Aversive aspects of a schedule of positive reinforcement. Journal of the Experimental Analysis of Behavior, 6 (3), 423-428. doi: 10.1901/jeab.1963.6-423

Azrin, N. H. (1961). Time out from positive reinforcement. Science, 8133, 382-383. doi: $10.1126 /$ science. 133.3450 .382

Azrin, N. H., Hake, D. F., \& Hutchinson, R. R. (1965). Elicitation of aggression by a physical blow. Journal of the Experimental Analysis of Behavior, 8(1), 55-58. doi: 10.1901/jeab.1965.8-55

Azrin, N. H., Hutchinson, R. R., \& Hake, D. F. (1963). Pain-induced fighting in the squirrel monkey. Journal of the Experimental Analysis of Behavior, 6(4), 620. doi: 10.1901/jeab.1963.6-620

Azrin, N. H., Hutchinson, R. R., \& Hake, D. F. (1966). Extinction-induced aggression. Journal of the Experimental Analysis of Behavior, 9, 191-204. doi: 10.1901/jeab.1966.9-191

Azrin, N. H., Hutchinson, R. R., \& McLaughlin, R. (1965). The opportunity for aggression as an operant reinforcer during aversive stimulation. Journal of the Experimental Analysis of Behavior, 8(3), 171-180. doi: 10.1901/jeab.1965.8-171 
Balsam, P., \& Bondy, F. (1983). The negative side effects of reward. Journal Applied Behavior Analysis, 3(16), 283-296. doi: 10.1901/jaba.1983.16-283

Banaco, R. A., \& Zagminani, D. R. (2018). Can clinical behavior analysis be conducted without aversive control? In J. C. Todorov (Ed.). Trends in Behavior Analysis, 3 (pp. 17-50). Brasília, DF: Technopolitik.

Baron, A., \& Galizio, M. (2005). Positive and negative reinforcement: Should the distinction be preserved? The Behavior Analyst, 28, 85-98. doi: 10.1007/bf03392107

Baron, A., \& Herpolsheimer, L. R. (1999). Averaging effects in the study of fixed-ratio response patterns. Journal of the Experimental Analysis of Behavior, 71(2), 145-153. doi: 10.1901/jeab.1999.71-145

Brown, T. G., \& Flory, R. K. (1972). Schedule-induced escape from fixed-interval reinforcement. Journal of the Experimental Analysis of Behavior, 17, 395-403. doi: 10.1901/jeab.1972.17-395

Campos, A. (2010). Análise de efeitos indesejáveis do reforço positivo (Dissertação de Mestrado). Universidade Estadual de Londrina, Londrina - PR, Brasil.

Catania, A. C. (1999). Aprendizagem: Comportamento, linguagem e cognição (4a. ed., D. G. de Souza et al., Trad.). Artmed, Porto Alegre. (Trabalho Original publicado em 1998).

Catania, A. C. (2008). The Journal of the Experimental Analysis of Behavior at zero, fifty, and one hundred. Journal of the Experimental Analysis of Behavior, 89, 111-118. doi: 10.1901/jeab.2008.89-111

Cherek, D. R., \& Heistad, G. T. (1971). Fixed-intervalinduced aggression. Psychonomic Science, 25, 7-8. doi: 10.3758/BF03335829

Critchfield, T. S. (2014). Skeptle's corner: Punishment Destructive or valuable social "adhesive"? Behavior Analysis Practice, 7, 36-44. doi: 10.1007/s40617-0140005-4

Cohen, P. S., \& Campagnoni, F. R. (1989). The nature and determinants of spatial retreat in the pigeon between periodic grain presentations. Animal Learning Behavior, 17, 39-48. doi: 10.3758/BF03205211

Dardano, J. F. (1973). Self-imposed timeouts under increasing response requirements. Journal of the Experimental Analysis of Behavior, 19(2), 269-287. doi: 10.1901/jeab.1973.19-269

Everly, J. B., Holtyn, A. F., \& Perone, M. (2014). Behavioral functions of stimuli signaling transitions across rich and lean schedules of reinforcement. Journal of the Experimental Analysis of Behavior, 101(2), 201-214. doi: 10.1002/jeab.74
Felton, M., \& Lyon, D. O. (1966). The post-reinforcement pause. Journal of the Experimental Analysis of Behavior, 9(2), 131-4. doi: 10.1901/jeab.1966.9-131

Ferster, C. B., \& Skinner, B. F. (1957). Schedules of reinforcement. Cambridge: Prentice Hall.

Gentry, W. D. (1968). Fixed-ratio schedule-induced aggression. Journal of the Experimental Analysis of Behavior, 11, 813-817. doi: 10.1901/jeab.1968.11-813

Guilhardi, H. (2016). Reforço Livre. Instituto de Terapia por Contingências de Reforçamento, Campinas - SP, Brasil. Recuperado de www.itcrcampinas.com.br/txt/reforcolivre.pdf

Griffiths, R. R., \& Thompson, T. (1973). The postreinforcement pause: A misnomer. The Psychological Record, 23, 229-235. doi: 10.1007/BF03394160

Harris, A., Foster, M.T., Levine, J., \& Temple, W. (2012). Effect of a signaled delay to reinforcement in the previous and upcoming ratios on between-ratio pausing in fixed ratio schedules. Journal of the Experimental Analysis of Behavior, 98, 295-309. doi: 10.1901/jeab.2012.98-295

Hineline, P. N. (1984). Aversive control: A separate domain? Journal of the Experimental Analysis of Behavior, 42, 495-509. doi: 10.1901/jeab.1984.42-495

Hunziker, M. H. L. (2011). Afinal, o que é controle aversivo? Acta Comportamentalia, 19, 9-19.

Hunziker, M. H. L. (2017). Dogmas sobre o controle aversivo. Acta Comportamentalia, 25(1), 85-100.

Knutson, J. F. (1970). Aggression during the fixed-ratio and extinction components of a multiple schedule of reinforcement. Journal of the Experimental Analysis of Behavior, 13, 221-231. doi: 10.1901/jeab.1970.13-221

Knutson, J. F., \& Kleinknecht, R. A. (1970). Attack during differential reinforcement of a low rate of responding. Psychonomic Science, 19, 289-290. doi: 10.3758/BF03328821

Leitenberg, H. (1965). Is time-out from positive reinforcement an aversive event? Psychological Bulletin, 64(6), 428-441. doi: 10.1037/h0022657

Lydersen, T. (1992). Timeout induced by differentialreinforcement-of-low-rate schedules. Behavioural Processes, 28, 1-12. doi: 10.1016/03766357(92)90044-E

Lydersen, T. (1997). Choice of timeout from fixed-time schedules: Comparison of two procedures. Behavioural Processes, 40, 137-147. doi: 10.1016/S03766357(96)00770-X

Michael, J. (1975). Positive and negative reinforcement: A distinction that is no longer necessary; or a better way to talk about bad things. Behaviorism, 3, 33-44. doi: 10.1300/J075v24n01_15 
Perone, M. (2003). Negative effects of positive reinforcement. The Behavior Analyst, 26(1), 1-14. doi: 10.1007/bf03392064

Perone, M. (2019). How I learned to stop worrying and love replication failures. Perspectives on Behavior Science, 42, 91-108. doi: 10.1007/s40614-018-0153-x

Perone, M., \& Courtney, K. (1992). Fixed-ratio pausing: Joint effects of past reinforcer magnitude and stimuli correlated with upcoming reinforcer magnitude. Journal of the Experimental Analysis of Behavior, 57, 33-46. doi: 10.1901/jeab.1992.57-33

Pitts, R. C., \& Malagodi, E. F. (1996). Effects of reinforcement amount on attack induced under a fixedinterval schedule in pigeons. Journal of the Experimental Analysis of Behavior, 65, 93-110. doi: 10.1901/jeab.1996.65-93

Powell, R. W. (1968). The effect of small sequential changes in fixed-ratio size upon the post-reinforcement pause. Journal of the Experimental Analysis of Behavior, 11, 589-593. doi: 10.1901/jeab.1968.11-589

Retzlaff, B. J., Parthum, E. T. P., Pitts, R. C., \& Hughes, C. E. (2017). Escape from rich-to-lean transitions: Stimulus change and timeout. Journal of the Experimental Analysis of Behavior, 107(1), 65-84. doi: 10.1002/jeab. 236

Rico, V. V., Carvalho Neto, M. B., Silveira, M. V. \& Barros, R. S. (2018). Aversive contingencies in Behavior Analysis: a survey of JEAB and JABA publications (1958-2018). Revista Brasileira de Análise do Comportamento, 14 (2), 199-206. doi: 10.18542/rebac.v14i2.7539

Sidman, M. (1989). Coercion and its fallout. Boston: Authors Cooperative.

Skinner, B.F. (1953). Science and human behavior. New York: MacMillan.

Skinner, B. F. (1971). Beyond freedom and dignity. Indianopolis: Hackett Publishing Company.

Skinner, B. F. (1974). Sobre o behaviorismo (trad. M. P. Villalobos,). São Paulo, SP: Cultrix.

Schlinger, H.D., Derenne, A., \& Baron, A. (2008). What 50 years of research tell us about pausing under ratio schedules of reinforcement. The Behavior Analyst, 31(1), 39-60. doi: 10.1007/BF03392160

Soares, P. F. R, \& Carvalho Neto, M. B. (2016). Agressão e análise do comportamento: A história do modelo de "pain-aggression". Revista Brasileira de Análise do Comportamento, 12, 65-74.

Soares, P. F. R., \& Goulart, P. R. K. (2015). Aggression in JEAB: A survey of publications. Revista Brasileira de Análise do Comportamento, 11, 93-100.

Thompson, D. M. (1964). Escape from $S^{\mathrm{D}}$ associated with fixed-ratio reinforcement. Journal of the Experimental Analysis of Behavior, 7, 1-8. doi: 10.1901/jeab.1964.7-1
Thompson, D. M. (1965). Time-out from fixed ratio reinforcement: A systematic replication. Psychonomic Science, 2, 109-110. doi: 10.3758/BF03343354

Ulrich, R. E. (1966). Pain as a cause of aggression. American Zoologist, 6, 643-662. doi: 10.1093/icb/6.4.643

Ulrich, R. E., \& Azrin, N. H. (1962). Reflexive fighting in response to aversive stimulation. Journal of Experimental Analysis of Behavior, 5(4), 511-520. doi: 10.1901/jeab.1962.5-511

Ulrich, R. E., Wolff, P. C., \& Azrin, N. H. (1964). Shock as an elicitor of intra-and inter-species fighting behavior. Animal Behavior, 12(1), 14-15. doi: 10.1016/0003-3472(64)90095-8

Wade-Galuska, T., Perone, M., \& Wirth, O. (2005). Effects of past and upcoming response-force requirements on fixed-ratio pausing. Behavioural Processes, 68, 91-95. doi: 10.1016/j.beproc.2004.10.001

Williams, D. C., Saunders, K. J., \& Perone, M. (2011). Extended pausing by humans on multiple fixed-ratio schedules with varied reinforcer magnitude and response requirements. Journal of the Experimental Analysis of Behavior, 95, 203-220. doi: 10.1901/jeab.2011.95-203

Young, R., Foster T. M., \& Bizo, L. A. (2017). The effects of reinforcer magnitude in the preceding and upcoming ratios on between-ratio pausing in multiple, mixed, and single fixed-ratio schedules. Journal of the Experimental Analysis of Behavior, 108(3), 414-432. doi: 10.1002/jeab.290

Zimmerman, J., \& Ferster, C. B. (1964). Some notes on time out from reinforcement. Journal of the Experimental Analysis of Behavior, 7(1), 13-19. doi: 10.1901/jeab.1964.7-13

Submetido em: 15/03/2019 Aceito em: 27/06/2019 